\title{
Risk of bleeding with direct oral anticoagulants versus warfarin: cohort studies using primary care datasets
}

Vinogradova Y, Coupland C, Hippisley-Cox. Risk of bleeding with direct oral anticoagulants versus warfarin: cohort studies using primary care datasets. British Journal of General Practice 2018; 68 (supplement 1): bjgp18X696629 doi: https://doi.org/10.3399/bjgp18X696629

Conference abstract available at: https://bjgp.org/content/68/suppl 1/bjgp18X696629

\begin{abstract}
Background

There is limited evidence on the safety of direct oral anticoagulants (DOACs) in real world settings.
\end{abstract}

Aim

To investigate risks of bleeding and all-cause mortality in DOACs compared to warfarin. Method

Prospective cohorts of incident oral anticoagulant users between 2011 and 2016 derived from two UK databases (QResearch and CPRD) linked to hospital and mortality data, with no anticoagulant prescriptions in the year before entry, were selected. Overall, 132,231 warfarin, 7744 dabigatran, 37,863 rivaroxaban, and 18,223 apixaban users, were sub-grouped into those with atrial-fibrillation (AF) and without (non-AF), followed up until any major bleed leading to hospital admission or death. Analyses were adjusted for demographics, life style, comorbidities and prescribed medications.

Results

Compared to warfarin, apixaban was associated with decreased risk of major bleeding (hazard ratio $0.62,95 \%$ confidence interval $[\mathrm{CI}]=0.54$ to 0.73 ), intracranial (HR 0.48, $95 \% \mathrm{CI}=0.34$ to 0.68 ) and gastro-intestinal (HR 0.67, 95\% CI $=0.54$ to 0.83 ).

Dabigatran and rivaroxaban were associated with decreased risk of intracranial bleeding (HR $0.44,95 \% \mathrm{CI}=0.27$ to $0.71 ; \mathrm{HR} 0.73,95 \% \mathrm{CI}=0.59$ to 0.90$)$. Rivaroxaban was associated with increased risk of all-cause mortality (HR $1.34,95 \% \mathrm{CI}=1.26$ to 1.42 ), consistent for both sub-cohorts. A small increased all-cause mortality risk for apixaban (HR $1.14,95 \% \mathrm{CI}=1.05$ to 1.24 ) was statistically significant on the lower doses (HR $1.31,95 \% \mathrm{CI}=1.19$ to 1.45 ) but not on higher doses (HR $0.98,95 \% \mathrm{CI}=0.87$ to 1.10).

\section{Conclusion}

Overall, apixaban was found to be the safest drug for any major bleed, particularly for patients with increased risk of intracranial or gastro-intestinal bleeding.

\section{Funding}

This work has been supported by NIHR with a SPCR round 11 grant [reference number 304]. We acknowledge the contribution of EMIS, CPRD, HES and Office of National Statistics for providing the data.

(C) British Journal of General Practice 2018 